\title{
Conversão catalítica do etanol sobre catalisadores suportados em ZSM-5
}

\section{(Catalytic conversion of ethanol over ZSM-5-supported catalysts)}

\author{
D. S. Lima, O. W. Perez-Lopez \\ Departamento de Engenharia Química, Universidade Federal do Rio Grande do Sul, R. Luiz Englert s/n, \\ Porto Alegre, RS, Brasil 90040-040 \\ perez@enq.ufrgs.br
}

\begin{abstract}
Resumo
O petróleo, fonte de energia não renovável, é a principal matéria-prima usada na produção de derivados como combustíveis, lubrificantes e petroquímicos básicos. Neste contexto o etanol, uma matéria-prima renovável, torna-se fonte para a obtenção de hidrocarbonetos de maior valor agregado, tais como, benzeno, tolueno, etc. Este trabalho teve como objetivo avaliar o efeito de diferentes metais suportados em zeólita HZSM-5 para a conversão do etanol em produtos com maior valor agregado. Duas séries de catalisadores foram preparadas com diferentes metais e diferentes teores de Ni em HZSM-5 comercial. As amostras foram caracterizadas por área específica $\mathrm{S}_{\mathrm{BET}}, \mathrm{DRX}, \mathrm{TPD}-\mathrm{NH}_{3}$ e TPO. Os ensaios de atividade foram realizados em um reator tubular de leito fixo na faixa de temperatura entre 300 e $400{ }^{\circ} \mathrm{C}$, usando etanol como reagente. Os resultados sugerem que o tipo e a quantidade de metal usado na modificação da zeólita HZSM-5 comercial influencia a seletividade para formação dos hidrocarbonetos superiores, bem como a temperatura de reação. A utilização de menores vazões favoreceu a formação de produtos pesados. A formação dos hidrocarbonetos superiores diminuiu ao longo da reação devido à deposição de carbono sobre a superfície do catalisador.

Palavras-chave: catálise heterogênea, conversão do etanol, zeólita HZSM-5, catalisadores suportados, hidrocarbonetos superiores.
\end{abstract}

\begin{abstract}
The oil, a non-renewable energy source, is the main raw material used to produce derivatives such as fuels, lubricants and petrochemicals. In this context, ethanol, a renewable feedstock, becomes a source for obtaining high value-added hydrocarbons, such as benzene, toluene, etc. This work aimed to evaluate the effect of different metal catalysts supported on HZSM-5 zeolite for the ethanol conversion into products with higher added value. Two series of catalysts were prepared with different metals and different amounts of Ni on commercial HZSM-5. Samples were characterized by specific area $S_{B E P}, X R D, N_{3}-T P D$ and TPO. The activity runs were carried out in a tubular fixed bed reactor in the temperature range between 300 and $400{ }^{\circ} \mathrm{C}$, using ethanol as a reactant. The results suggest that the type and the amount of metal used for the modification of commercial HZSM-5 zeolite influence the selectivity for formation of higher hydrocarbons as well as the reaction temperature. The formation of heavy products was favored by low flow rates and it was found that the selectivity for higher hydrocarbons declined throughout the reaction due to the deposition of carbon over the catalyst.
\end{abstract}

Keywords: heterogeneous catalysis, conversion of ethanol, HZSM-5 zeolite, supported catalysts, higher hydrocarbons.

\section{INTRODUÇÃO}

O petróleo tem sido responsável pelo fornecimento de um terço da energia primária consumida no planeta. Sua composição química, baseada em hidrocarbonetos de grande heterogeneidade molecular, abre caminhos para usos industriais mais especializados e sofisticados. Por isso, o petróleo é a principal matéria-prima empregada para produzir os derivados utilizados, tais como: combustíveis, lubrificantes e produtos petroquímicos [1]. Os compostos aromáticos são obtidos no processo de pirólise da nafta ou através da reforma catalítica (um dos processos envolvidos no refino de petróleo); sendo assim, a obtenção de hidrocarbonetos (em especial olefinas $\mathrm{C}_{3}-\mathrm{C}_{4}$ e aromáticos) através da conversão do etanol é uma importante alternativa e tem atraído considerável interesse já há algum tempo $[2,3]$.

A condição de seletividade e a acidez da zeólita ZSM-5 são adequadas para a transformação do etanol em eteno, olefinas $\mathrm{C}_{3}-\mathrm{C}_{4}$ ou em hidrocarbonetos [2, 4-7]. Também, observou-se, através de experimentos realizados para a reação de conversão de etanol, com diferentes metais impregnados sobre a zeólita ZSM-5, que o rendimento final de produtos BTX (benzeno, tolueno e xileno) é maior que no caso do uso da zeólita pura e, em alguns casos, a reação pode ser realizada a temperaturas relativamente baixas [8-12]. Van der Borght et al. [13] estudaram a conversão do etanol para a obtenção de hidrocarbonetos superiores utilizando HZSM-5 modificada com os metais $\mathrm{Fe}$, Ni e $\mathrm{Ga}$, onde os resultados foram comparados aos obtidos para a zeólita pura. Uma variedade de produtos foi detectada, incluindo olefinas leves, parafinas leves, aromáticos e uma fração pesada consistindo de hidrocarbonetos $\mathrm{C}_{5+}$ que são formados via conversão consecutiva do etileno. Phung et al. [14] estudaram a seletividade para os produtos na conversão do etanol, utilizando como catalisadores a zeólita HZSM-5 modificada com P, Fe e Ni, em diferentes teores. 
Segundo os autores a adição de $\mathrm{P}$ reduz significativamente a acidez e a atividade catalítica da HZSM-5, mas melhora a seletividade na formação do eteno. Além disso, diminui a formação de hidrocarbonetos superiores e a formação de coque. Para o Fe, foi observado que ocasiona uma diminuição na acidez da zeólita e, no caso da adição de Ni, diminui a acidez Brönsted, mas produz sítios ativos para a oligomerização levando à formação de mais produtos $\mathrm{C}_{4}$ e aromáticos. A conversão do etanol em hidrocarbonetos, particularmente olefinas leves e aromáticos, catalisada pela zeólita HZSM-5, sob diferentes condições de temperatura, pressão parcial do etanol e velocidade espacial, foi estudada em [15] e os resultados mostraram que os parâmetros estudados influenciaram significativamente no rendimento de propeno e aromáticos. Os autores sugerem que em menores temperaturas, a formação de éter etílico ocorreu via desidratação intermolecular, e então foi convertido em eteno. Por outro lado, em maiores temperaturas, a conversão direta de etanol em eteno ocorreu por desidratação intramolecular. Na sequência, a oligomerização do eteno leva à formação de olefinas mais pesadas, que podem ser convertidas por craqueamento em olefinas leves (propenos, butenos) ou ciclização e transferência de hidrogênio, formando aromáticos e parafinas.

A acidez e a estrutura porosa das zeólitas desempenham um papel muito importante na desativação dos catalisadores causada pelo coque, pois a formação de coque acontece em reações catalisadas por sítios ácidos localizados nos poros [16]. O efeito de desativação das moléculas de coque depende da força dos sítios ácidos, sendo os sítios ácidos mais fortes, aqueles que são mais ativos, os primeiros a desativarem [17]. Nesse contexto, o objetivo do presente trabalho é a preparação e avaliação de catalisadores suportados em HZSM-5 para aplicação na reação de conversão do etanol, visando a obtenção de hidrocarbonetos superiores.

\section{EXPERIMENTAL}

Preparação dos catalisadores: foram preparados dois grupos de catalisadores suportados em zeólita HZSM5 comercial fornecida pela Petrobrás: i) catalisadores impregnados com 2,5\% de diferentes metais e ii) catalisadores impregnados com diferentes teores de Ni. A impregnação dos metais sobre a zeólita HZSM-5 foi realizada com excesso de solvente, utilizando os nitratos desses metais como precursores, fixando-se em $1 \mathrm{~g}$ a massa de suporte utilizada para todas as amostras. A mistura foi mantida sob agitação constante à temperatura ambiente por um período de $4 \mathrm{~h}$, utilizando-se água destilada como solvente. O material foi seco em estufa a $80{ }^{\circ} \mathrm{C}$ durante $12 \mathrm{~h}$ e posteriormente calcinado a $600{ }^{\circ} \mathrm{C}$ durante $2 \mathrm{~h}$ com uma taxa de aquecimento de $10^{\circ} \mathrm{C} \cdot \mathrm{min}^{-1}$ e fluxo de ar sintético de $50 \mathrm{~mL} \cdot \mathrm{min}^{-1}$. A zeólita ZSM-5 pura, utilizada em testes para comparação, também foi calcinada utilizando as mesmas condições dos catalisadores impregnados.

Caracterização: a técnica de difratometria de raios $\mathrm{X}$ (DRX) foi utilizada para verificar a cristalinidade dos catalisadores impregnados em relação à ZSM-5 pura. Os difratogramas foram obtidos utilizando um difratômetro de raios $\mathrm{X}$ (Bruker, D2 Phaser), usando radiação $\mathrm{CuK} \alpha$. A área específica dos catalisadores foi obtida a partir de medidas de fisissorção de nitrogênio, utilizando o método dinâmico, com um único ponto. Previamente, as amostras foram submetidas a um pré-tratamento a $250^{\circ} \mathrm{C}$ por $30 \mathrm{~min}$, sob fluxo de 30 $\mathrm{mL} \cdot \mathrm{min}^{-1}$ de nitrogênio. A análise foi efetuada a $-196{ }^{\circ} \mathrm{C}$ em um sistema de análise multipropósito SAMP3 (Termolab Equipamentos), equipado com um detector de condutividade térmica (TCD), sob fluxo de mistura de $30 \%$ de $\mathrm{N}_{2}$ em hélio. $\mathrm{A}$ área específica das amostras foi determinada pelo método BET (Brunauer-Emmett-Teller). A acidez dos catalisadores foi determinada através de análises de dessorção à temperatura programada de amônia (TPD- $\mathrm{NH}_{3}$ ) no sistema de análise multipropósito SAMP3. Primeiramente, as amostras foram submetidas a um pré-tratamento a $100{ }^{\circ} \mathrm{C}$ com passagem de $30 \mathrm{~mL} \cdot \mathrm{min}^{-1}$ de hélio durante $30 \mathrm{~min}$. A etapa de adsorção de amônia foi realizada a $100{ }^{\circ} \mathrm{C}$, por $30 \mathrm{~min}$, utilizandose $30 \mathrm{~mL} \cdot \mathrm{min}^{-1}$ de mistura contendo 5 vol\% de amônia em hélio. Antes de se iniciar o aquecimento em si, foi realizada uma purga com passagem de hélio durante 30 min. Então, a temperatura foi elevada até $750^{\circ} \mathrm{C}$ com rampa de aquecimento de $10^{\circ} \mathrm{C} \cdot \mathrm{min}^{-1}$, sob o fluxo de hélio. A quantidade de carbono depositado nos catalisadores após a reação foi determinada através de ensaios de oxidação à temperatura programada (TPO), utilizando uma termobalança (TA Instruments, SDT Q600). Para isso, uma amostra de $10 \mathrm{mg}$ foi aquecida sob vazão de ar sintético de $100 \mathrm{~mL} \cdot \mathrm{min}^{-1}$ a uma taxa de $10{ }^{\circ} \mathrm{C} \cdot \mathrm{min}^{-1}$ até $800{ }^{\circ} \mathrm{C}$.

Ensaios de atividade catalítica: a atividade catalítica das amostras foi avaliada através da reação de conversão do etanol em um microrreator tubular de vidro, sendo nitrogênio alimentado no mesmo através de controlador de fluxo mássico (Sierra Instruments). Para a alimentação do etanol utilizou-se uma bomba dosadora tipo seringa (KD Scientific). O catalisador foi disposto em leito fixo, diluído com esferas de vidro de 32 a 42 mesh de diâmetro e sustentado por lã de quartzo. Os produtos foram analisados por cromatografia gasosa em linha (Varian $3600 \mathrm{cx}$ ), empregando-se nitrogênio como gás de arraste e uma coluna empacotada, Porapak Q, cuja temperatura foi mantida em $200{ }^{\circ} \mathrm{C}$. Foram utilizados detectores de ionização de chama (FID) e de condutividade térmica (TCD). Para o teste realizado com vazão de $0,4 \mathrm{~mL} \cdot \mathrm{h}^{-1}$, foram avaliadas as temperaturas na faixa de $300-400{ }^{\circ} \mathrm{C}$ em intervalos de $25^{\circ} \mathrm{C}$. Também, foi realizado um teste variando a vazão do etanol alimentado. Nesse teste as vazões empregadas foram de $0,2,0,3,0,4,0,8$ e $1,2 \mathrm{~mL} . \mathrm{h}$ ${ }^{1}$. Ainda, foi realizado um ensaio com duração de $10 \mathrm{~h}$ utilizando a vazão de $0,4 \mathrm{~mL} \cdot \mathrm{h}^{-1} \mathrm{e}$ a temperatura de $325^{\circ} \mathrm{C}$ para avaliar a estabilidade do catalisador $2,5 \mathrm{Ni} / \mathrm{ZSM}-5$. A identificação dos produtos formados foi realizada por GCMS utilizando um cromatógrafo a gás (Agilent, HP6890) equipado com detectores FID e MS e coluna HP-1. Para a análise dos resultados, os hidrocarbonetos produzidos na reação e analisados em linha por GC foram classificados 
Tabela I - Classificação dos produtos segundo o tempo de retenção na coluna cromatográfica.

[Table I - Product classification using the retention time in the chromatographic column.]

\begin{tabular}{cc}
\hline Produto & $\begin{array}{c}\text { Tempo de retenção } \\
(\mathrm{min})\end{array}$ \\
\hline Hidrocarbonetos leves & $\mathrm{t} \leq 1,3($ etanol) \\
Hidrocarbonetos intermediários & $1,3($ etanol $)<\mathrm{t} \leq 4$ \\
Hidrocarbonetos pesados & $\mathrm{t} \geq 4$ \\
\hline
\end{tabular}

em três grupos segundo seu tempo de retenção, como mostra a Tabela I.

\section{RESULTADOS E DISCUSSÃO}

\section{Caracterização dos catalisadores}

As Tabelas II e III apresentam os catalisadores preparados juntamente com sua composição nominal, a nomenclatura que foi adotada para cada uma das amostras e, também, os resultados obtidos para a área específica BET. Embora a área específica resultante do método BET com um único ponto não forneça os valores para o volume de microporos presentes na zeólita HZSM-5, esses dados foram

Tabela II - Catalisadores impregnados em HZSM-5 com diferentes metais.

[Table II - Samples impregnated with different metals and specific surface area.]

\begin{tabular}{cccc}
\hline Catalisador & $\begin{array}{c}\text { Metal } \\
\text { impregnado }\end{array}$ & $\begin{array}{c}\text { Teor de } \\
\text { metal }(\mathrm{wt} \%)\end{array}$ & $\begin{array}{c}\mathrm{S}_{\mathrm{BET}} \\
\left(\mathrm{m}^{2} / \mathrm{g}\right)\end{array}$ \\
\hline HZSM-5 & - & - & 370 \\
$2,5 \mathrm{Ni} / Z \mathrm{ZSM}-5$ & $\mathrm{Ni}$ & 2,5 & 328 \\
$2,5 \mathrm{La} / Z \mathrm{ZSM}-5$ & $\mathrm{La}$ & 2,5 & 343 \\
$2,5 \mathrm{Mg} / \mathrm{ZSM}-5$ & $\mathrm{Mg}$ & 2,5 & 289 \\
$2,5 \mathrm{Ca} / \mathrm{ZSM}-5$ & $\mathrm{Ca}$ & 2,5 & 308 \\
$2,5 \mathrm{Li} / Z \mathrm{ZSM}-5$ & $\mathrm{Li}$ & 2,5 & 226 \\
\hline
\end{tabular}

Tabela III - Catalisadores impregnados em HZSM-5 com diferentes teores de $\mathrm{Ni}$.

[Table III - Samples impregnated with different Ni contents and specific surface area.]

\begin{tabular}{cccc}
\hline Catalisador & $\begin{array}{c}\text { Teor de } \\
\mathrm{Ni}(\mathrm{wt} \%)\end{array}$ & $\begin{array}{c}\text { Catalisador } \\
\mathrm{S}_{\mathrm{BET}}\left(\mathrm{m}^{2} / \mathrm{g}\right)\end{array}$ & $\begin{array}{c}\text { Suporte } \\
\mathrm{S}_{\mathrm{BET}}\left(\mathrm{m}^{2} / \mathrm{g}\right)\end{array}$ \\
\hline $0,5 \mathrm{Ni} /$ ZSM-5 & 0,5 & 368 & 368 \\
1Ni/ZSM-5 & 1,0 & 332 & 366 \\
1,5Ni/ZSM-5 & 1,5 & 329 & 364 \\
2Ni/ZSM-5 & 2,0 & 328 & 363 \\
2,5Ni/ZSM-5 & 2,5 & 328 & 361 \\
3Ni/ZSM-5 & 3,0 & 326 & 359 \\
\hline
\end{tabular}

aqui utilizados no sentido comparativo. Pela Tabela II, notase que a impregnação da zeólita HZSM-5 com metais causa uma diminuição em sua área específica, sendo que o tipo de metal impregnado influencia nesse resultado. Alguns metais, como $\mathrm{Ni}$ e La, provavelmente ficaram mais bem dispersos na superfície da zeólita HZSM-5 do que os demais [18], que parecem ter se depositado principalmente nos poros do material, ocasionando um bloqueio de maior número destes.

A Tabela III apresenta os resultados obtidos para a área BET dos catalisadores preparados a partir da impregnação da HZSM-5 com diferentes teores de Ni. Observa-se que ocorre uma diminuição na área específica com o aumento do teor de níquel devido ao bloqueio parcial de poros da ZSM-5 pelas espécies de Ni [11, 12]. Segundo [19], a modificação com Ni provoca uma diminuição na área superficial da zeólita, devido a uma pequena diminuição no volume de microporos que é, provavelmente, decorrente do bloqueio parcial de alguns microporos pelo metal. Nota-se também que o efeito é maior para teores a partir de $1 \%$ de Ni. No entanto, se for considerada apenas a área específica correspondente ao suporte, isto é, descontando a quantidade de metal impregnado, observa-se que esta diminuição é muito menor (Tabela III). Além disso, nota-se também que a diferença entre a área do catalisador e a área do suporte permanece aproximadamente constante para as amostras contendo entre 1 e $3 \%$ de níquel.

A Fig. 1a mostra os resultados de TPD- $\mathrm{NH}_{3}$ para o suporte HZSM-5 e para os catalisadores obtidos através da impregnação da HZSM-5 com diferentes metais $(\mathrm{Ni}, \mathrm{La}, \mathrm{Mg}$, $\mathrm{Ca}$ e Li). A curva de TPD-NH3 da HZSM-5 mostra dois picos de dessorção, um em torno de $250{ }^{\circ} \mathrm{C}$ correspondente a sítios ácidos fracos e outro cujo máximo ocorre em aproximadamente $500{ }^{\circ} \mathrm{C}$ relativo a sítios ácidos fortes [20]. Notou-se que as amostras impregnadas com $\mathrm{Ni}$ e La foram aquelas que menos afetaram as propriedades ácidas da ZSM-5. Já nas amostras contendo $\mathrm{Mg}$ e Ca foram suprimidos os sítios ácidos fortes, possivelmente em virtude do caráter alcalino destes metais. Por outro lado, no catalisador contendo $\mathrm{Li}$ praticamente foram eliminadas as propriedades ácidas da ZSM-5, o que pode estar relacionado com o menor tamanho deste cátion indicando que o $\mathrm{Li}^{+}$se difundiu nos canais da zeólita ZSM-5, consequentemente, eliminando seus sítios ácidos. Este resultado está de acordo com o menor valor de área específica obtido para esta amostra (Tabela II). A deconvolução das curvas de TPD- $\mathrm{NH}_{3}$ utilizando uma função de distribuição Gaussiana indicou a existência de três tipos de sítios, os sítios ácidos fracos, representados pelo pico que aparece entre 200 e $300{ }^{\circ} \mathrm{C}$, os sítios de acidez moderada, que se encontram na faixa de $300-400{ }^{\circ} \mathrm{C}$ (na deconvolução), e os sítios de acidez forte, representados pelo pico que aparece entre 400 e $550{ }^{\circ} \mathrm{C}$.

A Fig. 1b juntamente com a Tabela IV apresentam os resultados de TPD- $\mathrm{NH}_{3}$ para os catalisadores impregnados com diferentes teores de Ni. Observa-se que a modificação da zeólita HZSM-5 com Ni ocasionou aumento nos sítios ácidos, que pode estar relacionado a uma interação do metal com a zeólita. Além disso, o pico relacionado à acidez moderada $\left(2^{\circ}\right.$ pico) aumentou para todas as amostras, enquanto que o pico relacionado à acidez forte $\left(3^{\circ}\right.$ pico) diminuiu, evidenciando 

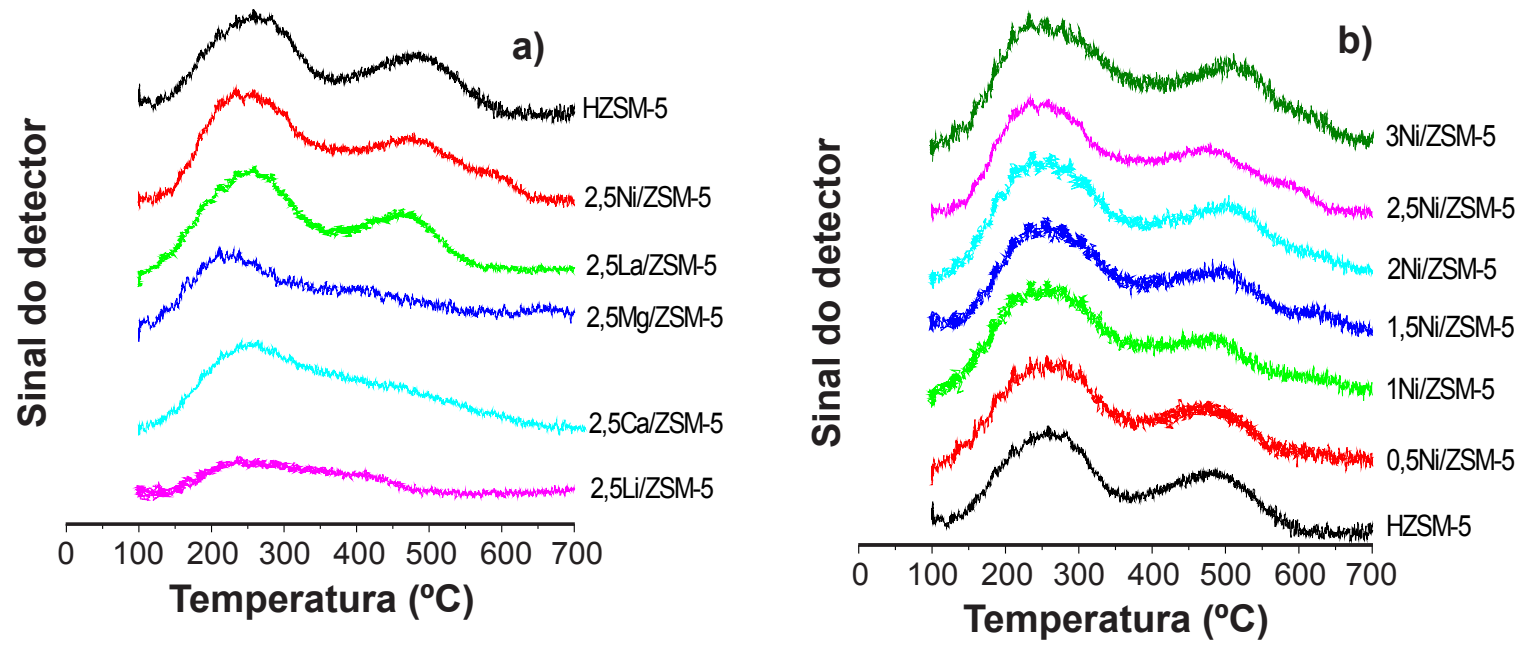

Figura 1: TPD-NH $\mathrm{NH}_{3}$ para os catalisadores modificados com: (a) diferentes metais; e (b) diferentes teores de Ni.

[Figure 1: NH3-TPD for modified catalysts with: (a) different metals; and (b) different amounts of Ni.]

Tabela IV - Deconvolução das curvas de TPD-NH $\mathrm{N}_{3}$ dos catalisadores Ni/ZSM-5.

[Table IV - Deconvolution of $\mathrm{NH}_{3}$-TPD curves for Ni/ZSM-5 catalysts.]

\begin{tabular}{|c|c|c|c|c|c|c|c|c|c|}
\hline \multirow{2}{*}{ Catalisador } & \multicolumn{4}{|c|}{ Temperatura $\left({ }^{\circ} \mathrm{C}\right)$} & \multicolumn{4}{|c|}{ Fração relativa ao total de sítios (\%) } & \multirow{2}{*}{$\begin{array}{c}\text { Total de sítios } \\
\text { ácidos (mmol/g cat) }\end{array}$} \\
\hline & $1^{\circ}$ pico & $2^{\circ}$ pico & $3^{\circ}$ pico & $4^{\circ}$ pico & $1^{\circ}$ pico & $2^{\circ}$ pico & $3^{\circ}$ pico & $4^{\circ}$ pico & \\
\hline HZSM-5 & 258 & 409 & 489 & - & 62,7 & 7,5 & 29,7 & - & 0,431 \\
\hline $0,5 \mathrm{Ni} / \mathrm{ZSM}-5$ & 249 & 420 & 472 & - & 49,9 & 38,1 & 12,0 & - & 0,526 \\
\hline 1Ni/ZSM-5 & 246 & 386 & 493 & - & 47,6 & 37,0 & 15,4 & - & 0,503 \\
\hline $1,5 \mathrm{Ni} / \mathrm{ZSM}-5$ & 247 & 354 & 495 & 633 & 45,7 & 23,8 & 28,5 & 7 & 0,493 \\
\hline 2Ni/ZSM-5 & 238 & 350 & 491 & 621 & 34,4 & 20,1 & 24,5 & 21 & 0,806 \\
\hline 2,5Ni/ZSM-5 & 228 & 311 & 455 & 535 & 31,1 & 31,8 & 16,1 & 21 & 0,630 \\
\hline $3 \mathrm{Ni} / \mathrm{ZSM}-5$ & 218 & 278 & 500 & 624 & 8,2 & 55,6 & 35,2 & 1 & 0,754 \\
\hline
\end{tabular}



Figura 2: Deconvolução da curva de TPD-NH 3 para o catalisador $2,5 \mathrm{Ni} / \mathrm{ZSM}-5$.

[Figure 2: Deconvolution of $\mathrm{NH}_{3}-\mathrm{TPD}$ curve for the catalyst $2.5 \mathrm{Ni} /$ ZSM-5.]

a diminuição da quantidade de sítios Brönsted [12], exceto para o 3Ni/ZSM-5. Estes resultados demonstraram que a modificação da zeólita HZSM-5 com Ni propicia aumento de acidez moderada [21]. Ainda, para as amostras com teores de Ni superiores a $1 \%$, surgiu um $4^{\circ}$ pico de dessorção em temperaturas maiores que $530{ }^{\circ} \mathrm{C}$, sendo mais pronunciado nas amostras 2Ni/ZSM-5 e 2,5Ni/ZSM-5 (Fig. 2). Esse resultado também foi observado em [14]. Analisando-se as temperaturas desses picos de dessorção, observou-se que para o catalisador que contém $2,5 \%$ de $\mathrm{Ni}$, ocorreu deslocamento dos mesmos para temperaturas mais baixas, indicando que a acidez dos sítios ativos foi levemente diminuída [22].

\section{Ensaios de atividade catalítica}

Testes com diferentes temperaturas de reação: a Tabela $\mathrm{V}$ apresenta um resumo dos resultados obtidos nos ensaios de atividade catalítica com diferentes temperaturas de reação para os catalisadores impregnados com diversos metais. Os resultados mostraram que, exceto a amostra 2,5Li/ZSM-5, todas as amostras levaram a $100 \%$ de conversão do etanol nos testes realizados entre 300 e $400{ }^{\circ} \mathrm{C}$. O catalisador contendo Li apresentou baixa atividade proporcionando uma conversão máxima de etanol de $13,8 \%$, na temperatura de $400{ }^{\circ} \mathrm{C}$. A baixa 
Tabela V - Conversão de etanol e seletividade para hidrocarbonetos pesados para catalisadores impregnados com diferentes metais. [Table V - Ethanol conversion and selectivity to heavy hydrocarbons to catalysts impregnated with different metals.]

\begin{tabular}{|c|c|c|c|c|c|}
\hline \multirow[b]{2}{*}{ Catalisador } & \multirow[b]{2}{*}{$\begin{array}{l}\text { Temperatura } \\
\left({ }^{\circ} \mathrm{C}\right)\end{array}$} & \multirow[b]{2}{*}{$\begin{array}{c}\text { Conversão do etanol } \\
(\%)\end{array}$} & \multicolumn{3}{|c|}{ Seletividade (\%) } \\
\hline & & & $\begin{array}{c}\mathrm{HC} \\
\text { pesados }\end{array}$ & $\begin{array}{l}\mathrm{HC} \\
\text { leves }\end{array}$ & $\begin{array}{c}\mathrm{HC} \\
\text { intermediários }\end{array}$ \\
\hline \multirow{2}{*}{ 2,5Ni/ZSM-5 } & 325 & 100 & 10,8 & 85,4 & 3,8 \\
\hline & 400 & 100 & 0,4 & 99,6 & 0 \\
\hline \multirow{2}{*}{ 2,5La/ZSM-5 } & 325 & 100 & 0 & 99,7 & 0,3 \\
\hline & 400 & 100 & 0,8 & 97,6 & 1,6 \\
\hline \multirow{2}{*}{ 2,5Mg/ZSM-5 } & 325 & 100 & 0 & 100 & 0 \\
\hline & 400 & 100 & 0 & 99,9 & 0,1 \\
\hline \multirow{2}{*}{ 2,5Ca/ZSM-5 } & 325 & 100 & 0 & 100 & 0 \\
\hline & 400 & 100 & 0 & 99,5 & 0,5 \\
\hline \multirow{2}{*}{ 2,5Li/ZSM-5 } & 325 & 1,9 & 0 & 76,4 & 23,6 \\
\hline & 400 & 13,8 & 0 & 96,3 & 3,7 \\
\hline
\end{tabular}
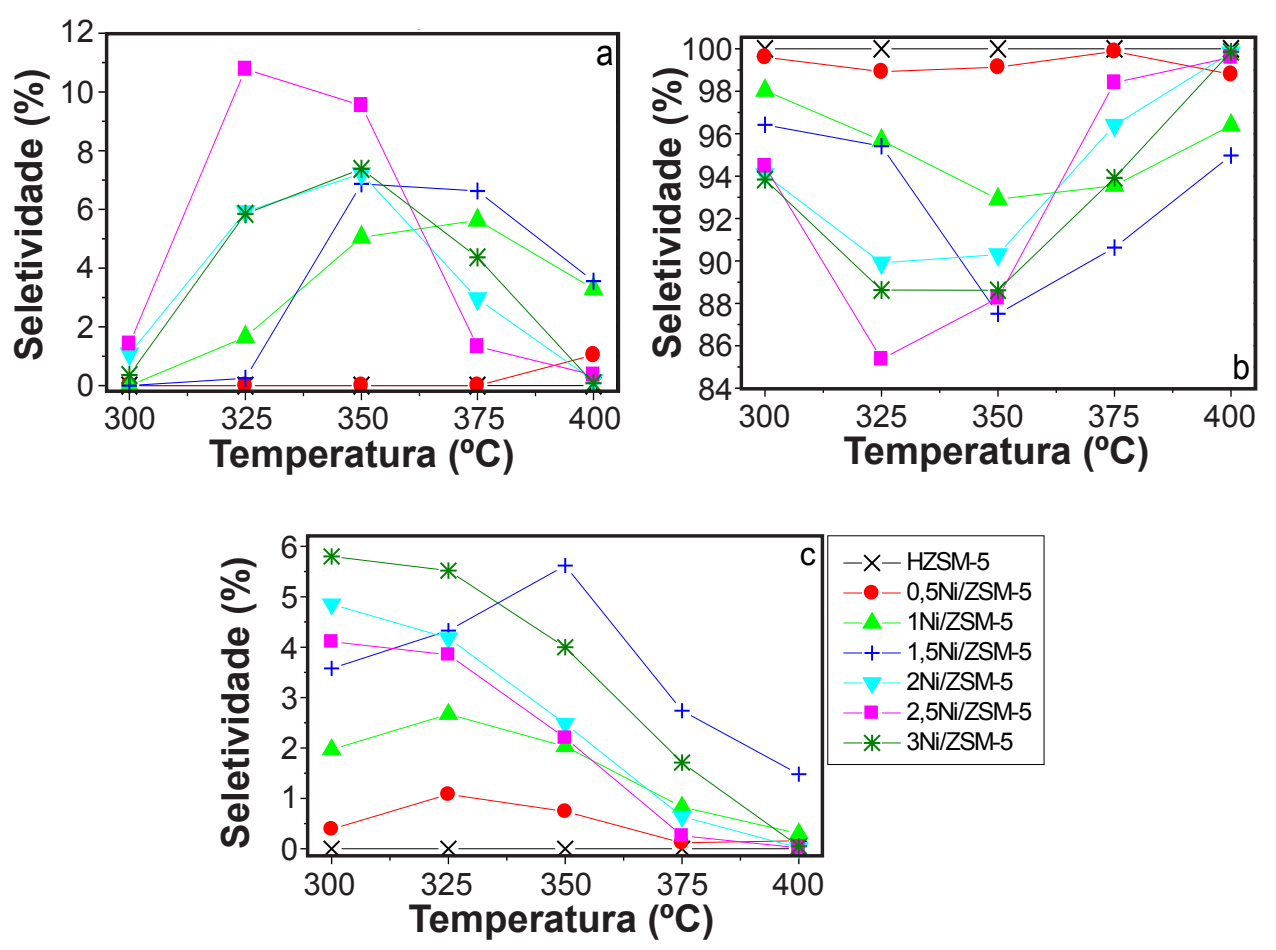

Figura 3: Produtos da conversão do etanol sobre catalisadores Ni/ZSM-5: a) hidrocarbonetos pesados, b) hidrocarbonetos leves e c) hidrocarbonetos intermediários.

[Figure 3: Conversion products from ethanol on Ni/ZSM-5 catalysts: a) heavy hydrocarbons, b) light hydrocarbons, and c) intermediary hydrocarbons.]

atividade da amostra 2,5Li/ZSM-5 foi relacionada com o fato do Li ter destruído as propriedades ácidas da HZSM-5, conforme mostraram os resultados de TPD-NH $\mathrm{NH}_{3}$ (Fig. 1a). Além disso, notou-se que os catalisadores com propriedades alcalinas foram pouco seletivos para os hidrocarbonetos superiores quando comparados ao catalisador 2,5Ni/ZSM-5. Isso ocorreu devido à alteração nas propriedades ácidas da ZSM-5, verificada pela TPD- $\mathrm{NH}_{3}$, já que a formação dos hidrocarbonetos superiores aparentemente requer sítios ácidos fortes. Dentre os catalisadores contendo metais com propriedades alcalinas, aquele preparado com La, que apresentou menor influência na acidez e menor redução de área específica, foi o único que apresentou seletividade para hidrocarbonetos superiores, na temperatura de $400{ }^{\circ} \mathrm{C}$. Isso pode ser devido ao fato de que a adição de La à zeólita ZSM-5 inibe a formação de coque, melhorando a seletividade para a formação dos compostos aromáticos [18, 23].

Para os catalisadores com diferentes teores de $\mathrm{Ni}$, os 
resultados dos testes realizados entre 300 e $400{ }^{\circ} \mathrm{C}$ levaram à completa conversão do etanol para todas as amostras. A Fig. 3 apresenta a seletividade em hidrocarbonetos pesados, leves e intermediários em função da temperatura da reação. A Fig. 3a mostra que o suporte (HZSM-5) não apresentou seletividade para hidrocarbonetos superiores. A seletividade para a formação de hidrocarbonetos superiores aumentou conforme aumentou o teor de $\mathrm{Ni}$ impregnado atingindo o maior valor para o catalisador contendo 2,5\% de $\mathrm{Ni}$ na temperatura de reação de 325 ${ }^{\circ} \mathrm{C}$. No entanto, um aumento subsequente do teor do $\mathrm{Ni}$ até 3\% resultou em uma diminuição na seletividade para a formação dos hidrocarbonetos pesados, o que pode ser decorrência da aglomeração do Ni na superfície da zeólita ao invés de sua dispersão. Para os catalisadores com menores teores de $\mathrm{Ni}$, notou-se que são necessárias maiores temperaturas de reação para que ocorra a formação dos produtos pesados. A Fig. 3b, que mostra a seletividade para hidrocarbonetos leves, apresenta um comportamento oposto ao de hidrocarbonetos pesados (Fig. 3a), isto é, quanto menor a seletividade para eteno maior a seletividade para hidrocarbonetos pesados, indicando que o eteno, produto predominante entre os leves, é um intermediário para a formação dos hidrocarbonetos superiores [24]. O catalisador 2,5Ni/ZSM-5 que apresentou maior seletividade para os hidrocarbonetos pesados a $325^{\circ} \mathrm{C}$ mostrou a menor seletividade na formação dos hidrocarbonetos leves, na mesma temperatura. Notou-se também que a ZSM-5 pura utilizada como suporte formou apenas produtos leves com $100 \%$ de seletividade, principalmente eteno. Quanto à formação dos hidrocarbonetos intermediários (Fig. 3c), a seletividade para a formação destes produtos aumenta na faixa de temperatura de $325-350{ }^{\circ} \mathrm{C}$, para os catalisadores com menores teores de Ni. Já no caso dos catalisadores com maiores teores de $\mathrm{Ni}$, a seletividade para esses produtos diminuiu conforme aumentou a temperatura de reação.

Influência do tempo de residência: a Fig. 4a apresenta os resultados para o teste realizado com temperatura fixa $\left(325^{\circ} \mathrm{C}\right)$ e vazão de etanol variável, para avaliar a influência do tempo de residência na formação dos produtos empregando-se o catalisador 2,5Ni/ZSM-5. Para todas as vazões testadas, obtiveram-se $100 \%$ de conversão do etanol e, quanto à seletividade, esse teste mostrou que para tempos de residência intermediários, isto é, para vazões relativamente baixas de etanol, a formação dos hidrocarbonetos superiores é favorecida, sendo o melhor
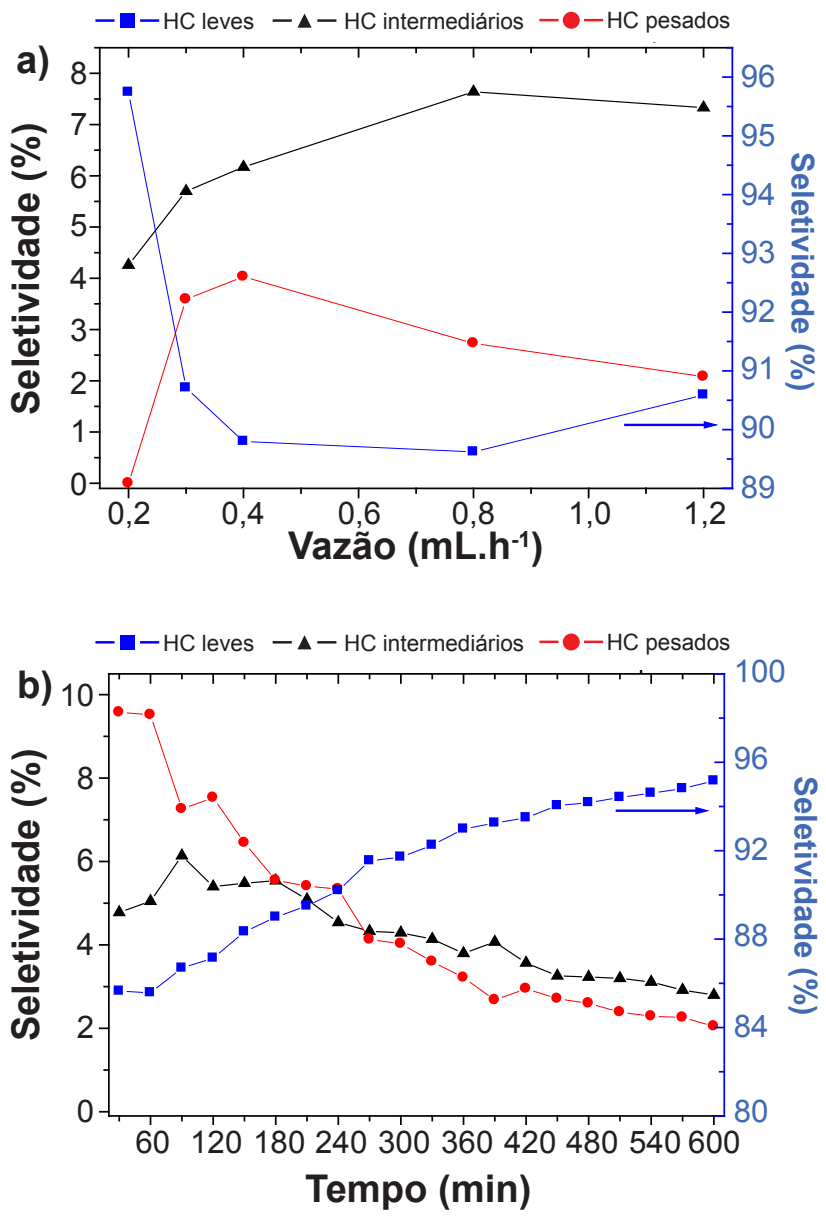

Figura 4: Influência do tempo de residência na seletividade (a) e produtos formados ao longo do teste de estabilidade (b) do catalisador $2,5 \mathrm{Ni} / \mathrm{ZSM}-5\left(\mathrm{~T}=325^{\circ} \mathrm{C}\right)$.

[Figure 4: Influence of the residence time on the selectivity (a) and products formed along the stability test (b) for 2.5Ni/ZSM-5 catalyst $\left(T=325^{\circ} \mathrm{C}\right)$.]

resultado obtido para a vazão de $0,4 \mathrm{~mL} \cdot \mathrm{h}^{-1}$. No caso de altas vazões, a seletividade para os hidrocarbonetos pesados diminuiu devido ao tempo de contato entre reagente e catalisador ser muito curto. Por outro lado, uma vazão de reagente muito baixa resultou também em uma diminuição da seletividade para hidrocarbonetos superiores, indicando neste caso a decomposição dos compostos pesados em compostos mais leves para tempos de residência muito longos, que é o que ocorreu para a vazão de $0,2 \mathrm{~mL} \cdot \mathrm{h}^{-1}$. Estes resultados mostraram a necessidade de um tempo de

Tabela VI - Produtos formados na decomposição catalítica do etanol.

[Table VI - Products formed in the catalytic decomposition of ethanol.]

\section{Leves}

Hidrocarbonetos
Metano, etano, eteno, propeno, isobutano, n-butano, trans-2-buteno, 1-buteno, isobuteno, cis-2-buteno

Intermediários

1-penteno, etilciclopropano

Pesados 2-metil-buteno-1, 2-metil-buteno2, 2-metil-pentano, 1,1 -dimetilciclopropano 
residência adequado no qual é favorecida a formação de produtos pesados.

Teste de estabilidade dos catalisadores: utilizando as condições que favorecem a obtenção de hidrocarbonetos superiores $\left(\mathrm{T}=325{ }^{\circ} \mathrm{C}\right.$ e vazão $\left.=0,4 \mathrm{~mL} \cdot \mathrm{h}^{-1}\right)$, foi realizado o teste de estabilidade (Fig. 4b) para verificar o comportamento da atividade e da seletividade do catalisador 2,5Ni/ZSM-5 durante a reação. Esse teste mostrou que ao longo da reação ocorreu diminuição na seletividade para formação dos hidrocarbonetos superiores e intermediários devido à formação de carbono (como mostra a análise de TPO apresentada abaixo), que vai se depositando sobre a superfície do catalisador bloqueando seus poros, consequentemente aumenta a seletividade para a formação dos hidrocarbonetos leves. Quanto à estabilidade, ao final das $10 \mathrm{~h}$ de reação ainda se obteve completa conversão do etanol. A Tabela VI traz os resultados da análise qualitativa dos produtos formados durante o teste de estabilidade. Observou-se a formação de hidrocarbonetos e compostos oxigenados, entre eles o propeno que é um produto de alto valor agregado e tem grande importância para a indústria petroquímica, por isso sua obtenção a partir de recursos renováveis tem atraído muita atenção nos últimos anos [25]. Quanto aos hidrocarbonetos pesados, obtiveram-se produtos de importantes usos na indústria, tais como, os butenos na obtenção de elastômeros e o metilpentano na fabricação de solventes.

\section{Caracterização dos catalisadores após a reação}

Os resultados de análises de oxidação à temperatura programada (TPO) para os catalisadores preparados a partir dos metais $\mathrm{Ni}, \mathrm{Mg}, \mathrm{Ca}, \mathrm{Li}$ e $\mathrm{La}$ suportados em zeólita HZSM-5 são apresentados na Fig. 5a, juntamente com a Tabela VII. Verificou-se menor perda de massa para os catalisadores $\mathrm{La}, \mathrm{Mg}$ e $\mathrm{Li}$, quando comparados ao catalisador 2,5Ni/ZSM-5, que apresentou maior acidez, sendo que esta foi a única amostra a apresentar um pico de oxidação de carbono em temperaturas acima de $400{ }^{\circ} \mathrm{C}$. Este resultado deve-se a esses materiais apresentarem propriedades alcalinas enquanto que a formação de coque é favorecida em superfícies ácidas. No caso da amostra contendo $\mathrm{Ca}$, que apresentou maior perda de massa, principalmente nos primeiros $200{ }^{\circ} \mathrm{C}$ do aquecimento, esse resultado pode estar relacionado à perda de umidade e/ou compostos voláteis adsorvidos.

A Fig. 5b juntamente com a Tabela VIII apresentam os resultados de TPO para os catalisadores impregnados com diferentes teores de $\mathrm{Ni}$. Estes resultados indicaram maior formação de coque durante a reação para a HZSM-5 pura, em relação àquelas modificados com $\mathrm{Ni}$, devido à maior acidez forte deste material, evidenciando que a formação de coque é favorecida quanto maior for a quantidade de sítios ácidos do material. Analisando somente os catalisadores impregnados com $\mathrm{Ni}$, observou-se que para maiores teores de metal, houve maior perda de massa, demonstrando maior formação de coque; isto ocorreu porque esses catalisadores
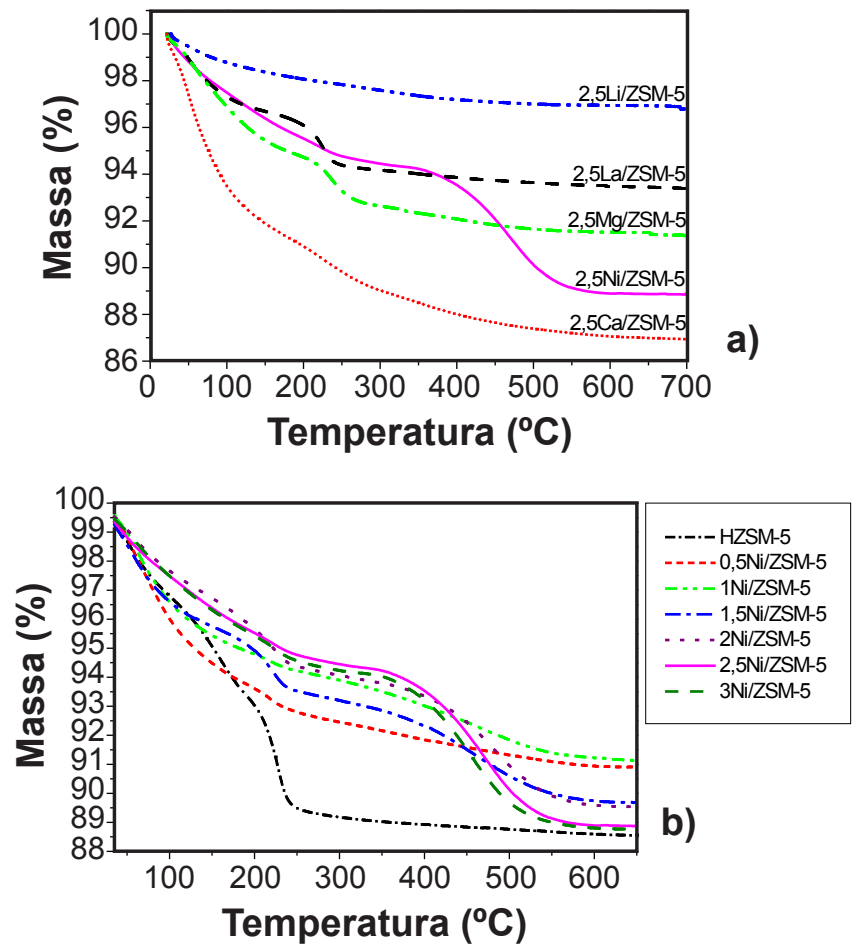

Figura 5: TPO dos catalisadores preparados com: (a) metais diversos; e (b) diferentes teores de $\mathrm{Ni}$.

[Figure 5: TPO of the catalysts prepared with: (a) different metals; and $(b)$ different Ni contents.]

foram aqueles que proporcionaram maior seletividade para a formação dos produtos pesados, sendo que a obtenção desses produtos requer sítios ácidos mais fortes [26].

Azeólita pura, mais ácida, e os catalisadores impregnados com menores teores de $\mathrm{Ni}$ apresentaram perda de massa na faixa de temperatura de $225-230{ }^{\circ} \mathrm{C}$, indicando que o carbono formado foi oxidado em temperaturas mais amenas, sendo, portanto, do tipo amorfo. A formação de depósitos de carbono amorfo reflete a formação de coque não catalítico, resultante de reações que envolvem como intermediários íons de carbono formados na superfície dos catalisadores em sítios ácidos de Brönsted ou Lewis [27]. Já os catalisadores impregnados com maiores teores de $\mathrm{Ni}$ mostraram perda de massa no intervalo de $350-500{ }^{\circ} \mathrm{C}$, demonstrando que

Tabela VII - Resultados de TPO para os catalisadores preparados a partir de metais diversos.

[Table VII - TPO results for the catalysts prepared with different metals.]

\begin{tabular}{|c|c|c|c|c|c|}
\hline \multirow{2}{*}{ Amostra } & \multicolumn{3}{|c|}{ Perda de massa $(\%)$} & \multicolumn{2}{|c|}{$\begin{array}{l}\text { Temperatura } \\
\text { DTA }\left({ }^{\circ} \mathrm{C}\right)\end{array}$} \\
\hline & $<200^{\circ} \mathrm{C}$ & $200-700{ }^{\circ} \mathrm{C}$ & Total & $1^{\circ}$ pico & $2^{\circ}$ pico \\
\hline $2,5 \mathrm{Ni} / \mathrm{ZSM}-5$ & 4,5 & 6,6 & 11,1 & 227 & 475 \\
\hline 2,5La/ZSM-5 & 3,9 & 2,7 & 6,6 & 225 & - \\
\hline 2,5Mg/ZSM-5 & 5,3 & 3,3 & 8,6 & 240 & - \\
\hline $2,5 \mathrm{Ca} / \mathrm{ZSM}-5$ & 9,1 & 4,0 & 13,1 & 235 & - \\
\hline 2,5Li/ZSM-5 & 1,9 & 1,3 & 3,2 & 320 & - \\
\hline
\end{tabular}


Tabela VIII - Resultados de TPO para os catalisadores preparados a partir de diferentes teores de $\mathrm{Ni}$.

[Table VIII - TPO results for catalysts prepared with different Ni contents.]

\begin{tabular}{cccccc}
\hline \multirow{2}{*}{ Amostra } & \multicolumn{3}{c}{ Perda de massa $(\%)$} & \multicolumn{3}{c}{ Temperatura } \\
& $<200{ }^{\circ} \mathrm{C}$ & $200-700{ }^{\circ} \mathrm{C}$ & Total & $1^{\circ}$ pico & $\left.2^{\circ} \mathrm{C}\right)$ \\
& pico \\
\hline HZSM-5 & 7,0 & 4,4 & 11,4 & 230 & - \\
$0,5 \mathrm{Ni} /$ ZSM-5 & 6,4 & 2,8 & 9,2 & 218 & - \\
$1 \mathrm{Ni} / \mathrm{ZSM}-5$ & 5,2 & 3,7 & 8,9 & 221 & 460 \\
$1,5 \mathrm{Ni} /$ ZSM-5 & 5,1 & 5,3 & 10,4 & 225 & 460 \\
$2 \mathrm{Ni} / \mathrm{ZSM}-5$ & 4,3 & 6,2 & 10,6 & 224 & 485 \\
$2,5 \mathrm{Ni} / \mathrm{ZSM}-5$ & 4,5 & 6,6 & 11,1 & 227 & 475 \\
$3 \mathrm{Ni} / \mathrm{ZSM}-5$ & 4,6 & 6,8 & 11,3 & 228 & 460 \\
\hline
\end{tabular}

nesses casos o carbono ou coque formado é do tipo mais duro. Isso se deve ao fato de que catalisadores metálicos promovem a adsorção dissociativa e a desidrogenação de hidrocarbonetos, originando depósitos de carbono, por vezes de elevada cristalinidade e maior estabilidade com morfologia que pode se apresentar como filamentos, com partículas metálicas associadas, em geral no topo, por vezes ao longo do filamento. Segundo [27], pode-se idealizar o seguinte mecanismo reacional para a formação catalítica de carbono filamentar: i) o hidrocarboneto é adsorvido e dissociado na superfície metálica, com formação de átomos de carbono adsorvidos; ii) estes átomos de carbono podem se dissolver e difundir através do metal, precipitando em regiões de crescimento preferencial, como as fronteiras de grão ou as interfaces metal/suporte. Desta forma, as partículas metálicas são destacadas da superfície e transportadas com os filamentos em crescimento, enquanto que a superfície ativa continua disponível para a reação já que o carbono não se acumula sobre ela; iii) é também possível a nucleação na superfície, conduzindo à formação de filmes de carbono que efetivamente desativam o catalisador, o chamado carbono encapsulante, responsável pela perda de atividade do catalisador ao longo da reação catalítica. A Fig. 6 apresenta

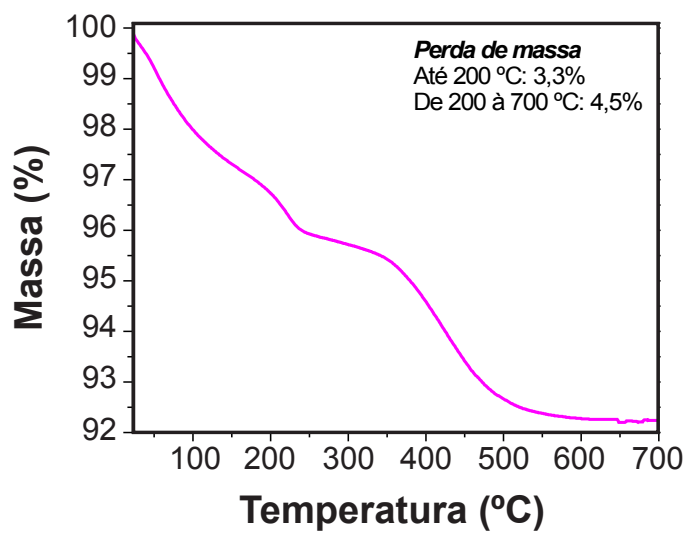

Figura 6: TPO do catalisador 2,5Ni/ZSM-5 utilizado no teste de estabilidade.

[Figure 6: TPO of the 2.5Ni/ZSM-5 catalyst used in the stability test.] a perda de massa decorrente durante a análise de TPO para a amostra 2,5Ni/ZSM-5 utilizada no teste de estabilidade. Observou-se que essa amostra apresentou a formação de dois tipos de carbono: o carbono amorfo, identificado pela perda de massa na faixa de $200-250{ }^{\circ} \mathrm{C}$, e o carbono mais duro, indicado pela perda de massa entre $350-500{ }^{\circ} \mathrm{C}$.

\section{CONCLUSÕES}

A modificação com metais da zeólita HZSM-5 causa diminuição na área específica. As análises de TPD- $\mathrm{NH}_{3}$ demonstraram que a modificação da zeólita HZSM-5 com diferentes metais altera as propriedades ácidas do suporte. A maior influência foi observada para as amostras impregnadas com $\mathrm{Mg}, \mathrm{Ca}$ e $\mathrm{Li}$, diminuindo significativamente os sítios ácidos do catalisador. Para a modificação da zeólita HZSM-5 com diferentes teores de $\mathrm{Ni}$, observou-se aumento de acidez moderada do suporte. Nos ensaios de atividade, com exceção da amostra 2,5Li/ZSM-5, todos os demais catalisadores preparados levaram a $100 \%$ de conversão do etanol. Os testes de atividade catalítica em diferentes temperaturas de reação sugerem que o tipo de metal e a quantidade impregnada influenciam na seletividade para formação dos hidrocarbonetos superiores, bem como a temperatura de reação. Dentre os metais avaliados, o catalisador impregnado com $\mathrm{Ni}$ foi o que apresentou melhor desempenho na conversão do etanol em hidrocarbonetos pesados. Para os catalisadores com diferentes teores de Ni o melhor resultado de seletividade para os hidrocarbonetos superiores foi obtido na temperatura de $325{ }^{\circ} \mathrm{C}$ para o catalisador impregnado com 2,5\% (2,5Ni/ZSM-5). O teste de avaliação do tempo de residência na formação dos produtos mostrou que a formação de hidrocarbonetos pesados é favorecida em maiores tempos de residência, isto é, para menores vazões de etanol. O teste de estabilidade mostrou que a seletividade para hidrocarbonetos pesados diminui ao longo da reação, enquanto a seletividade para hidrocarbonetos leves aumenta, em razão da formação de coque sobre a superfície do catalisador e, dentre os produtos formados, destaca-se o propeno que tem alto valor agregado. As análises de TPO realizadas após os ensaios catalíticos demonstraram que materiais com maior acidez e aqueles que tiveram melhores desempenhos na formação dos hidrocarbonetos superiores apresentam maior formação de carbono.

\section{AGRADECIMENTO}

À CAPES pelo apoio financeiro.

\section{REFERÊNCIAS}

[1] A.D. Quelhas, C.N. Passos, D.F.S. Lage, E. Abadie, E.C.M. Sousa, F.G. Cordeiro, I.M. Krahl, M.A. Farah, M.A.S. Araújo, N.I. Brasil, P.R.C. Camargo, R.R.C. Pinto, Processamento de petróleo e gás: petróleo e seus derivados, processamento primário, processos de refino, petroquímica, meio ambiente, LTC, Rio de Janeiro, Brasil (2011). 
[2] A.G. Gayubo, A. Alonso, B. Valle, A.T. Aguayo, J. Bilbao, Appl. Catal. B 97 (2010) 299.

[3] Y.I. Makarfi, M.S. Yakimova, A.S. Lermontov, V.I. Erofeev, L.M. Koval, V.F. Tretiyakov, Chem. Eng. J. 154 (2009) 396.

[4] R. Barthos, A. Széchenyi, F. Solymosi, J. Phys. Chem. B 110 (2006) 21816.

[5] Y. Furumoto, Y. Harada, N. Tsunoji, A. Takahashi, T. Fujitani, T. Ide, M. Sadakane, T. Sano, Appl. Catal. A 399 (2011) 262.

[6] M. Inaba, K. Murata, M. Saito, I. Takahara, React. Kinet. Catal. Lett. 88 (2006) 135.

[7] A.M. Varvarin, K.M. Khomenko, V.V. Brei, Fuel 106 (2013) 617.

[8] N.R. Machado, V. Calsavara, N.G. Astrath, A.M. Neto, M.L. Baesso, Appl. Catal. A 311 (2006) 193.

[9] N.R. Machado, V. Calsavara, N.G. Astrath, C.K. Matsuda, A.P. Junior, M.L. Baesso, Fuel 84 (2005) 2064.

[10] B.M. Nichele, O.W. Perez-Lopez, Anais do $14^{\circ}$ Congresso Brasileiro de Catálise, Porto de Galinhas, PE (2007).

[11] A.G. Gayubo, A. Alonso, B. Valle, A.T. Aguayo, M. Olazar, J. Bilbao, Fuel 89 (2010) 3365.

[12] X. Zhang, J. Zhong, J. Wang, L. Zhang, J. Gao, A. Liu, Fuel Process. Technol. 90 (2009) 863.

[13] K. Van Der Borght, V.V. Galvita, G.B. Marin, Appl. Catal. A 492 (2015) 117.

[14] T.K. Phung, R. Radikapratama, G. Garbarino, A. Lagazzo, P. Riani, G. Busca, Fuel Process. Technol. 137
(2015) 290.

[15] Z.S.B. Sousa, C.O. Veloso, C.A. Henriques, V.T.J. Da Silva, Mol. Catal. A-Chem. 422 (2016) 266.

[16] F.F. Madeira, K.B. Tayeb, L. Pinard, H. Vezin, S. Maury, N. Cadran, Appl. Catal. A 443 (2012) 171.

[17] M. Guinsnet, F.R. Ribeiro, Zeólitos: um nanomundo ao serviço da catálise, Fund. Calouste Gulbenkian, Lisboa, Portugal (2004).

[18] Y. Ni, A. Sun, X. Wu, J. Hu, T. Li, G. Li, Chin. J. Chem. Eng. 19, 3 (2011) 439.

[19] A.G. Gayubo, A. Alonso, B. Valle, A.T. Aguayo, M. Olazar, J. Bilbao, Chem. Eng. J. 167 (2011) 262.

[20] S. Masiero, N. Marcilio, O.W. Perez-Lopez, Catal. Lett. 131 (2009) 194.

[21] B. Valle, A. Alonso, A. Atutxa, A.G. Gayubo, J. Bilbao, Catal. Today 106 (2005) 118.

[22] A. Takahashi, W. Xia, I. Nakamura, H. Shimada, T. Fujitani, Appl. Catal. A. 423-424 (2012) 162.

[23] Y. Ni, W. Peng, A. Sun, W. Mo, J. Hu, T. Li, G. Li, J. Ind. Eng. Chem. 16, 4 (2010) 503.

[24] A.T. Aguayo, A.G. Gayubo, A.M. Tarrio, A. Atutxa, J. Bilbao, J. Chem. Technol. Biotechnol. 77 (2002) 211.

[25] D. Sun, Y. Yamada, S. Sato, Appl. Catal. B 174-175 (2015) 13.

[26] F.F. Madeira, N.S. Gnep, P. Magnoux, H. Vezin, S. Maury, N. Cadran, Chem. Eng. J. 161 (2010) 403.

[27] M.H. Herbst, M.I.F. Macêdo, A.M. Rocco, Quim. Nova 27, 6 (2004) 986.

(Rec. 04/08/2016, Rev. 07/10/2016, 21/12/2016, Ac. $11 / 03 / 2017)$ 